\title{
Negative refraction through an extraordinary transmission left-handed metamaterial slab
}

\author{
M. Beruete, ${ }^{1}$ M. Navarro-Cía, ${ }^{1}$ M. Sorolla, ${ }^{1}$ and I. Campillo ${ }^{2}$ \\ ${ }^{1}$ Millimeter and Terahertz. Waves Laboratory, Universidad Pública de Navarra, E-31006 Pamplona, Spain \\ ${ }^{2}$ CIC-nanoGune Consolider, Tolosa Hirbidea 76, 20018 Donostia, Spain
}

(Received 17 January 2008; revised manuscript received 8 April 2009; published 8 May 2009)

\begin{abstract}
In this work we present numerical and experimental results of negative refraction through a negative index metamaterial based on miniaturized extraordinary transmission stacked subwavelength hole arrays, also known as stacked fishnet structures, working in the millimeter wave range. Analytical transmittance as a function of frequency and tangential momentum shows the conditions under which negative refraction is expected. Due to the role of the gap-surface-plasmon-polariton-like mode that gives rise to the resonant magnetic response of this structure, strong dispersion with the angle of incidence is expected. Experimental results using a quasioptical bench in the millimeter wave band demonstrate negative refraction and, besides, reasonable agreement with the simulation results is obtained. A discussion based on leaky waves serves to qualitatively explain the main features. The presented results may find application in the design of new lenses and devices both in the microwave, terahertz, and optical ranges.
\end{abstract}

DOI: 10.1103/PhysRevB.79.195107

PACS number(s): 41.20.Jb, 42.25.-p, 42.70.Qs, 78.20.Ci

Left-handed metamaterials (LHM) or negative refractive index media (NIM) allow the possibility of reversing Snell's law, i.e., to obtain negative refraction at the interface between a standard medium and a NIM. That means that light is bent anomalously, crossing the normal. ${ }^{1,2}$ Negative index of refraction occurs when both dielectric permittivity and magnetic permeability are simultaneously negative. Also, in a NIM, phase and group velocities have opposite signs.

An experimental measurement of NIM was given in Ref. 3 by mixing a wire medium $(\varepsilon<0)$ (Ref. 4) and an array of split-ring resonators (SRRs) $(\mu<0){ }^{5}$ Then, a wedge-shaped geometry was constructed, i.e., a prism, showing negative refraction. Negative index has also been reported in photonic crystals. $^{6,7}$

In turn, extraordinary optical transmission (EOT) (Ref. 8) is a high transmittance peak that appears in the hole cut-off region of perforated metallic plates. This phenomenon was initially ascribed to the coupling of light to surface plasmons, but the replica under perfect electric conductor (PEC) formalism theoretically ${ }^{9}$ and experimentally in other regions of the electromagnetic spectrum ${ }^{10}$ showed that plasmons are not necessarily involved in the process (see Refs. 11 and 12).

By simply stacking EOT hole arrays, left-handed propagation is obtained in the longitudinal dimension when the period is subwavelength, allowing to use the metamaterial terminology in that dimension. In Ref. 13 we measured an extraordinary transmission metamaterial (ETM) in the millimeter wave range that was constructed following that method. Similar results, but outside the scope of EOT, were presented at optical frequencies ${ }^{14,15}$ where plate alignment difficulties and losses for such tiny holes restricted the stacking to a pair of fishnet plates for the optical arrangements. A proposal to bypass these limitations has been recently proposed by using semiconductor layers and avoiding resonances (see Ref. 16). In Ref. 13 we analyzed longitudinal LH propagation by using an inverse transmission line, with the parallel inductance given by the cutoff holes and the series capacitance by the electric coupling between correlative plates. Also, the role of evanescent modes in an equivalent waveguide analysis was highlighted in Ref. 17. Furthermore, a miniaturized design was presented in Ref. 18 .

In a recent work, we experimentally observed negative refraction through an ETM wedge constructed with double periodic subwavelength hole arrays. ${ }^{19}$ In this structure, there is left-handed propagation uniquely along the longitudinal direction. Due to the staircase wedge approximation, whose step is determined by the lateral dimension $d_{x}$ of the unit cell and longitudinal LH propagation, adjacent holes located at the output face show a negative phase difference which causes the desired negative refraction.

In this work, we experimentally demonstrate negative refraction through an ETM slab with parallel faces, i.e., with no special shape. This result is essentially different from the previously-mentioned negative refraction through a prism. Here, negative refraction is intrinsic to the structure and comes up as a result of LH propagation in two axes, longitudinal and transversal. The negative refraction under oblique incidence at ETM is experimentally reported. This paper is mainly devoted to experimental results that can be complemented with a recently reported theoretical analysis. ${ }^{20}$

The structure under consideration along this paper is depicted in Fig. 1: a doubly-periodic dielectric-loaded extraordinary transmission subwavelength hole array. The dimensions of the structure are the same as those employed in Ref. 18, namely: hole array periods, $3.4 \mathrm{~mm}$ and $1.5 \mathrm{~mm}$; stack period, $0.525 \mathrm{~mm}$; hole diameter, $1.2 \mathrm{~mm}$; substrate parameters: relative dielectric constant, $\varepsilon_{r}=2.43$; dielectric thickness, $h=0.49 \mathrm{~mm}$; metallization thickness, $t=35 \mu \mathrm{m}$; conductivity, $\sigma=5.8 \cdot 10^{7} \mathrm{~S} / \mathrm{m}$; and circular wafer diameter, 62.4 $\mathrm{mm}$. With these parameters, an extraordinary transmission band is expected within the $V$ band of the millimeter wave spectrum. $^{18}$

The experiment was carried out employing a single wafer and four stacked wafers. In our notation, a single wafer consists of a metallic perforated plate sandwiched between two identical dielectric slabs, with a total thickness of around $1.015 \mathrm{~mm}(=t+2 h)$. The stack of four wafers has a total thickness of $2.59 \mathrm{~mm}(=4 t+5 h)$.

The measurements were done using an $\mathrm{ABmm}^{\mathrm{TM}}$ Vector Network Analyzer and a quasioptical bench (see Fig. 2). The 


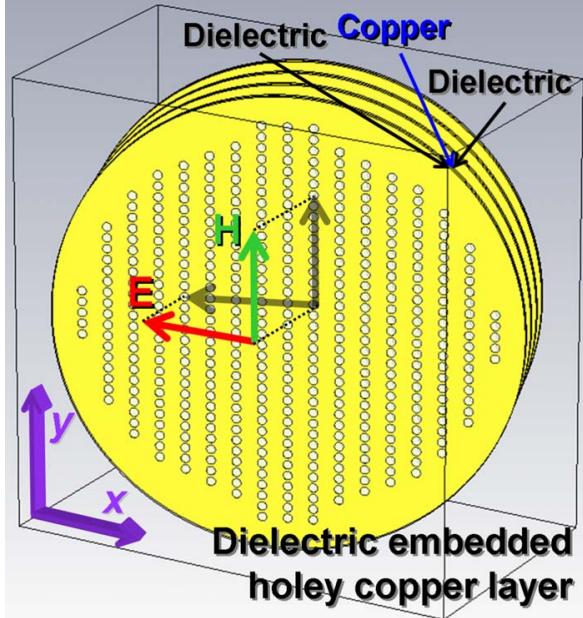

FIG. 1. (Color online) Schematic of four stacked wafers. The wafers have been moved away from each other for clarity. Parameters: short period, $1.5 \mathrm{~mm}$; large period, $3.4 \mathrm{~mm}$; stack period, $0.525 \mathrm{~mm}$; and hole diameter, $1.2 \mathrm{~mm}$. Substrate parameters: dielectric constant, $\varepsilon_{r}=2.43$; thickness, $0.49 \mathrm{~mm}$; metallization thickness, $35 \mu \mathrm{m}$; and conductivity, $\sigma=5.8 \cdot 10^{7} \mathrm{~S} / \mathrm{m}$. Circular wafer diameter, $62.4 \mathrm{~mm}$.

elliptical mirrors provide a collimated Gaussian beam illumination $^{21}$ on the sample location. As depicted, the plane of incidence is the $x-z$ plane and the polarization used is $P$, i.e., the electric field is parallel to the plane of incidence. Several angles of incidence were studied by rotating the samples around the $y$ axis. Notice that with the polarization used, the tangential component of the electric field $\left(E_{t}\right)$ is parallel to the large hole periodicity, a necessary condition to excite EOT resonance. ${ }^{18}$

We studied experimentally the frequency dependence and beam deflection for one and four stacked wafers as a function of the angle of incidence. We considered five different

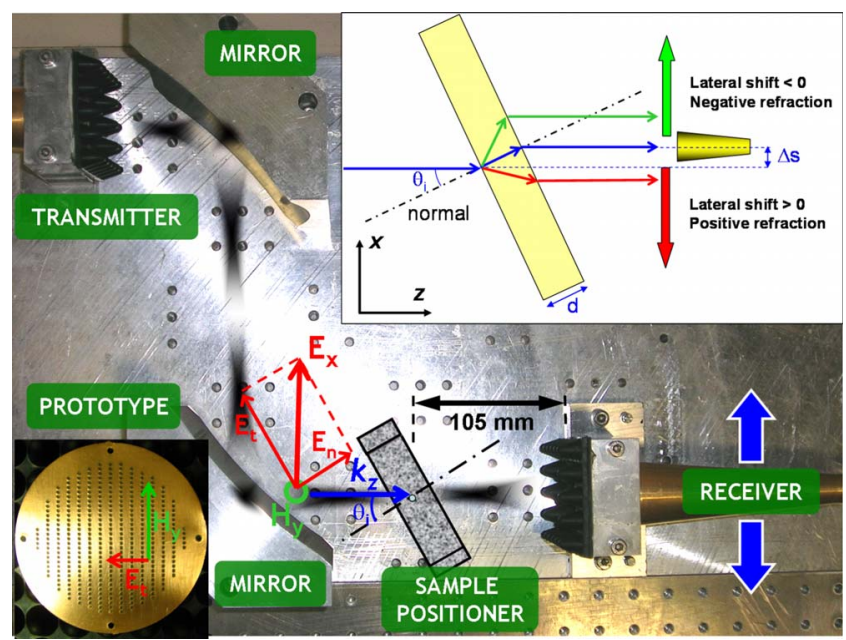

FIG. 2. (Color online) Photograph of the setup comprising twohorn antennas (transmitter and receiver), a pair of elliptical mirrors to focus the beam on the sample under test, and (inset on the left) a picture of the fabricated prototype with the orientation of the incident electromagnetic fields. angles, namely $\theta_{i}=0,5^{\circ}, 10^{\circ}, 20^{\circ}$, and $30^{\circ}$. To characterize the beam deflection we performed a scan of the power distribution at the output with the receiver horn antenna. A sketch of the setup is depicted schematically in the top inset of Fig. 2. Notice that the origin of the lateral shift was placed at the location where the transmitted ray would emerge if $n$ $=0$, that is, following the normal, to correlate unambiguously negative beam displacement with negative refraction. This position corresponds to a lateral shift $\Delta s=d \sin \left(\theta_{i}\right)$ with respect to the location of the incident beam, where $d$ is the total thickness of the slab and $\theta_{i}$ is the angle of incidence. This is our reference of the lateral displacement $L$. Following the notation of the figure, if the maximum of the transmitted beam is found at a place where $L$ is positive, then it can be said that the beam is positively refracted. Conversely if the maximum is found at a negative $L$, then the beam was negatively refracted, or the bulk material is a NIM.

The measurement results of a single wafer are plotted in Fig. 3(a). First, we show the free-space measurement i.e., no sample in the path between antennas, to test the correctness of the experimental setup (labeled in Fig. 3 as Gaussian beam). Then, we introduce the sample under test with a single wafer and check the normal-incidence behavior. A clear frequency filtering effect is obtained within the EOT band (around $67 \mathrm{GHz}$ ). No beam deflection is noticed but some collimation is observed compared to the unperturbed Gaussian beam when no sample is introduced. Anyway, the beam width at the output is similar to the free-space measurement. Increasing the angle of incidence to $\theta_{i}=5^{\circ}$ has a clear effect in the lateral beam displacement, which progresses slightly toward negative values. The frequency filtering is also present, and the band remains approximately within the above-mentioned frequencies. An increment to $\theta_{i}=10 \mathrm{deg}$, emphasizes the negative displacement and also moves the frequency band downwards. Notice also that the peak amplitude diminishes somewhat, but still has an appreciable level. At the incidence angle of $\theta_{i}=20 \mathrm{deg}$, the lateral shift remains approximately unchanged but the frequency band is clearly shifted and appears between 58 and $62 \mathrm{GHz}$. Also, a second band emerges at the end of the band $(71 \mathrm{GHz})$ and seemingly no lateral shift appears. Finally, when $\theta_{i}$ $=30 \mathrm{deg}$, the first band is located between 52 and $56 \mathrm{GHz}$ with practically the same beam shift and a reduced but still noticeable peak level, about $-9 \mathrm{~dB}$. The second band is also shifted downwards in frequency with negligible lateral shift. The measured frequency toward lower frequencies for a single EOT wafer is in good agreement with the analysis shown in Ref. 22.

When four wafers are stacked, the measurement under normal incidence shows a frequency filtering with first and second EOT peaks located around 54.6 and $60.5 \mathrm{GHz}$, respectively (see again Ref. 18). Due to losses, the peak level in both cases is around $-15 \mathrm{~dB}$, a moderately high value. When $\theta_{i}=5 \mathrm{deg}$, the EOT band (formed by both aforementioned peaks) maintains its position and a clear negative beam shift of $L=5 \mathrm{~mm}$ appears. In the case of $\theta_{i}=10 \mathrm{deg}$, the band downshifts to peak frequencies of 54.2 and 58.3 $\mathrm{GHz}$ with a lateral beam displacement of $L=20 \mathrm{~mm}$ and 10 $\mathrm{mm}$, correspondingly. Very interestingly, when $\theta_{i}=20 \mathrm{deg}$, transmission is abruptly destroyed indicating that the critical 

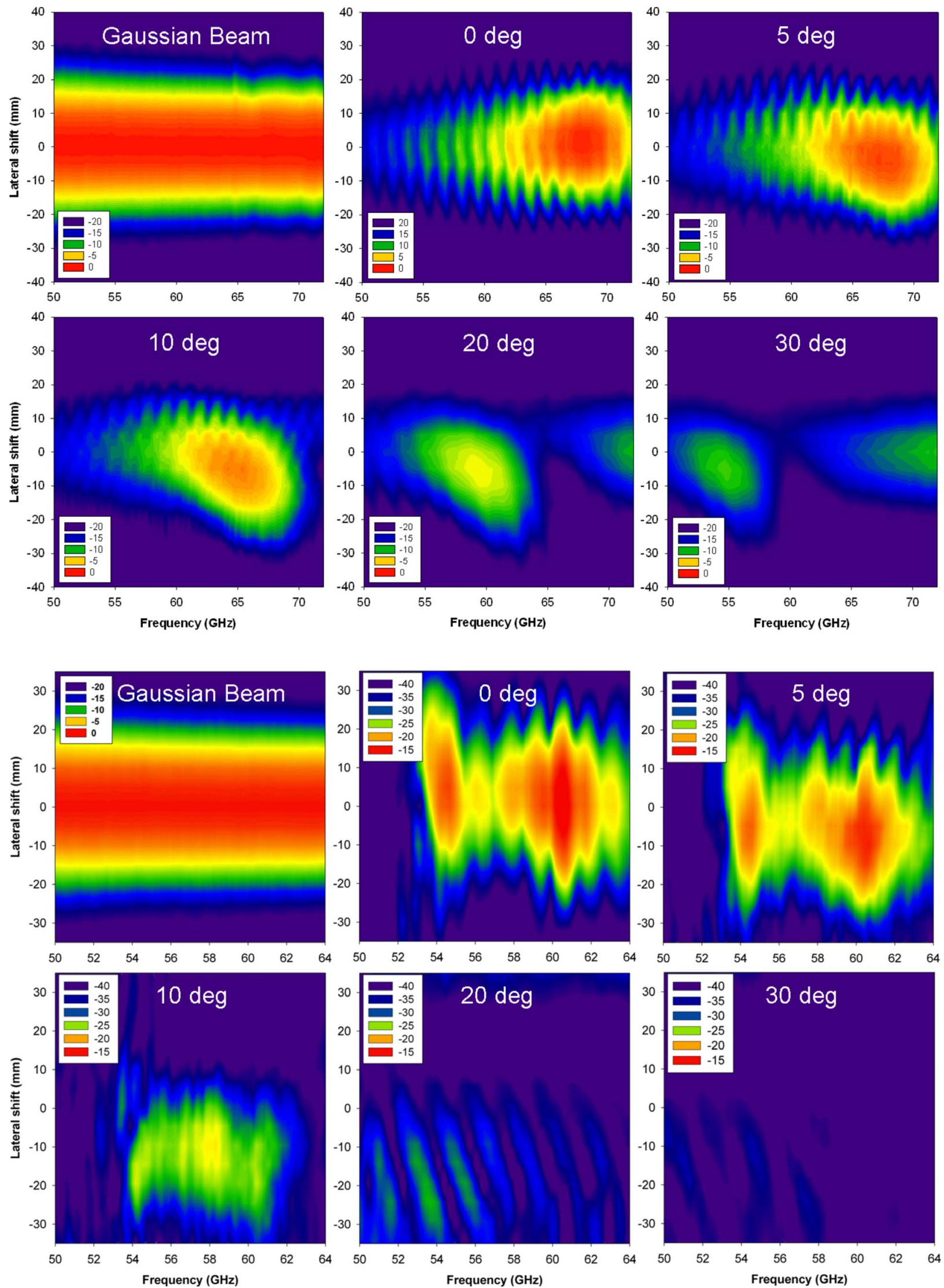

FIG. 3. (Color online) Experimental magnitude of the transmission coefficient of a single wafer (a) and four stacked wafers (b) measured when the incident wave has its polarization contained on the $x-z$ plane, that is the $P$ polarization. Data are collected at five different angles: $0^{\circ}$ (normal incidence), $5^{\circ}, 10^{\circ}, 20^{\circ}$, and $30^{\circ}$. Note that there are two different transmittance scales for clarity. 


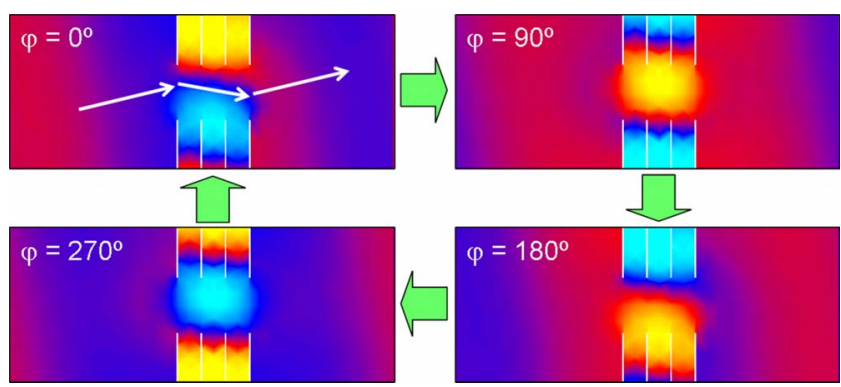

FIG. 4. (Color online) Time evolution of the magnetic field $H_{y}$ in the $x-z$ plane for four equispaced phases $\left(\varphi=0,90^{\circ}, 180^{\circ}\right.$, and $270^{\circ}$ ).

angle to observe transmission is close to this value. Finally, at $\theta_{i}=30 \mathrm{deg}$, transmission is completely annihilated. These results are in concordance with the theoretical analysis of Ref. 20.

From the experimental results, a negative beam deflection (negative refraction) is observed both for a single and four stacked wafers, but it is clearly enhanced when a bulk material is constructed by stacking wafers as it can be easily concluded from the fact that the wave propagates more distance for the case of four stacked plates than for the isolated one. The Gaussian beam suffers considerable displacements to negative values, as large as $L=-20 \mathrm{~mm}(4 \lambda)$ when $\theta_{i}$ $=10 \mathrm{deg}$. Clearly, as the angle of incidence increases, the beam displacement also increases, but also losses are enlarged and when $\theta_{i}=30 \mathrm{deg}$ the transmission is practically negligible. The results here presented are in good agreement with the analytical study of one wafer and two stacked wafers shown in Ref. 20 [two-dimensional (2D) hole array and double fishnet, respectively, according to notation in this reference].

To reinforce the experimental results we have simulated the case of four stacked wafers when $\theta_{i}=10 \mathrm{deg}$ with the commercial solver CST Microwave Studio (CST MWS). ${ }^{23}$ Periodic boundary conditions have been imposed on the contour, giving as a result a semi-infinite structure. The plot of Fig. 4 shows the time evolution of $H_{y}$ at a cross section where the hole can be easily identified. With the polarization we are using, $H_{y}$ is the total magnetic field. Negative refraction is evident in the figure as stressed by the arrows. Moreover, attending to the evolution of the field along the $x$ axis, it is observed that outside the structure the maximum evolves toward positive values, whereas inside the structure, it evolves toward negative values.

The key to discern between positive and negative refraction is to determine the relative sign of the tangential group and phase velocity inside the material: if both have the same sign, then positive refraction takes place; on the other hand, if they have different signs, then negative refraction happens. This has been schematically depicted in Fig. 5. It is observed that both in positive and negative refraction, the tangential wave vector must be conserved at the interface, as it is well known. Therefore, to get negative refraction, the tangential group velocity must reverse and so the relative sign of phase and group velocities is different. In the standard positive refraction, both have the same sign.
Negative Refraction

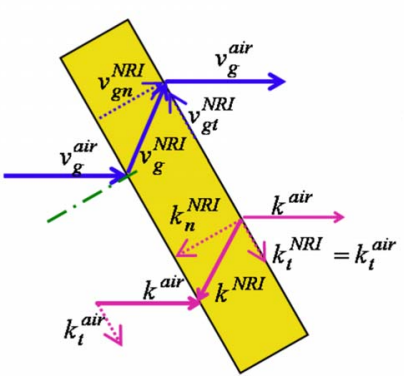

\section{Positive Refraction}

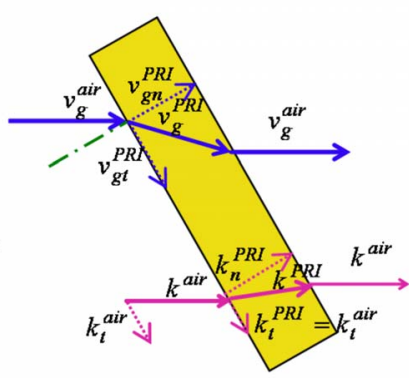

FIG. 5. (Color online) Representation of the mechanism of negative (left) and positive refraction (right) through a slab under oblique incidence illumination. To guide the eyes, blue (upper) and pink (lower) arrows have been used to represent group velocity and wave vector, respectively, in both cases.

Using the frequency domain solver of CST MWS we have simulated the transmittance through four semi-infinite stacked wafers. We performed a parameter sweep of the tangential phase difference (normalized in Fig. 6 as $k_{t} d_{t} / 2 \pi$ ) and found the transmittance in every case. In the vertical axis of Fig. 6 is depicted the normalized frequency $d_{t} / \lambda$.

Following the previous discussion to determine negative refraction, one has to compare the relative propagation sense of phase and group velocities. Both velocities can be extracted from the dispersion diagram taking into account that the direction of the average phase velocity is parallel to $k$ and the direction of the average group velocity is given by $\operatorname{grad}_{\mathbf{k}}(\omega){ }^{7,24}$ Then, the group velocity is proportional to the slope of the band depicted in Fig. 6.

On the one hand, several modes are excited in ETM according to our analytical angular dependence of the transmission. The additional resonant modes respect to one

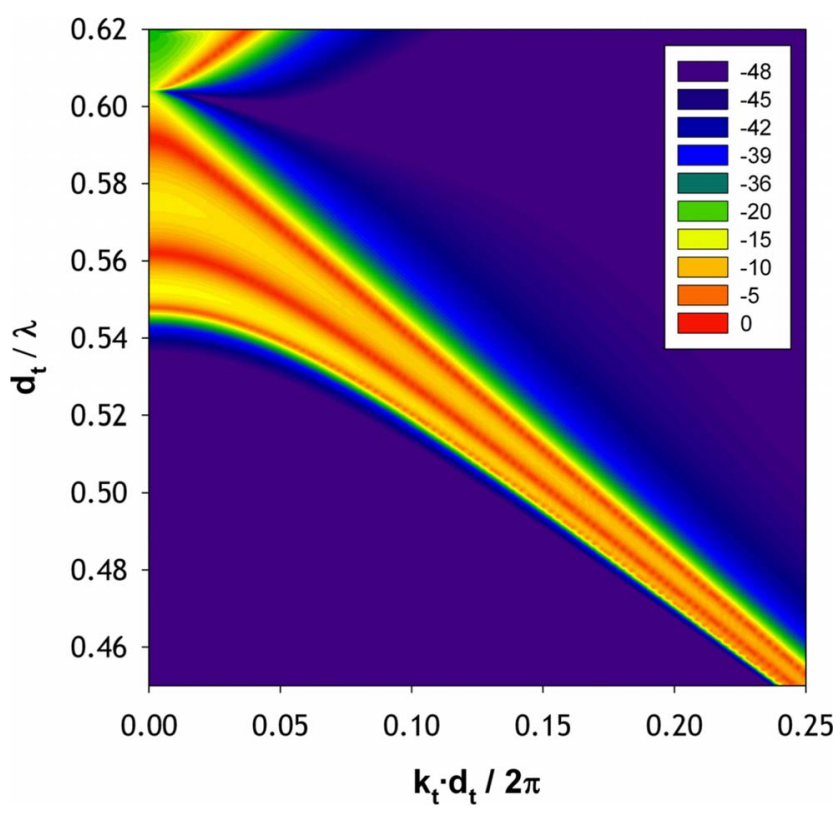

FIG. 6. (Color online) Simulated angular dependence of the transmission particularized to the first band of four stacked subwavelength hole arrays for $P$-polarization incidence. 
extraordinary- transmission-perforated metallic plate have been correlated with the symmetric and antisymmetric modes that come as a result of electromagnetic coupling through the hole pattern between neighboring spoof gapsurface plasmon polariton. ${ }^{25,26}$ On the other hand, it is plainly seen that the first band formed by three of the aforementioned hybridized modes has a negative slope. Then, the tangential group velocity is negative, whereas the tangential phase velocity is positive. So, negative refraction is also foreseen in the simulation. From previous works we know that also the normal components are antiparallel (see Ref. 18) and in consequence, we can affirm that the material has negative characteristics in both axes.

This transversal infinite structure results can serve to qualitatively predict the behavior of realistic and finite structures. First, let us consider angles close to the normal incidence $\left(k_{t} \approx 0\right)$ : in this region, the group velocity has a predominant longitudinal component ( $z$ component), which leads to negligible lateral displacement as a result of an almost normal transmission. Then, as the angle of incidence increases, the slope of the curve becomes more significant, i.e., transversal component of the group velocity is emphasized which makes the transmitted beam move away from the normal, causing a measurable lateral displacement. Finally when the angle of incidence is large enough, the tangential group-velocity vector crosses a long fraction of the slab which theoretically would produce a very large lateral displacement for the infinite structure whereas in the practical finite structure case, results in negligible transmission, i.e., a critical angle beyond which no transmission is achieved. This agrees qualitatively with the measurements, where it is observed that the peak of transmission decreases as the angle of incidence is enlarged. Moreover, also the shrinkage of the band as the angle of incidence increases agrees well with experiment and analytical study. Logically, nothing beyond a qualitative agreement can be expected, since in the infinite structure simulation the peculiar features of the finite counterpart are lost. For instance, the finite number of holes can introduce an additional penalty in the transmission coefficient as the angle of incidence moves away from the normal, since the illumination is less efficient.

As explained in Refs. 12 and 18, EOT phenomena are directly related to the coupling of the incident and transmitted fields to leaky waves. More specifically the incident wave is coupled to the $m=-1$ space harmonic that propagates along the surface and is reradiated again at the output At the EOT frequency, this space harmonic has antiparallel phase and group velocities, or in other words, it has a LH propagation characteristic. Thus, the combination of longitudinal and transversal left-handed propagation effects makes possible the achievement of negative refraction by stacking EOT hole arrays.

Summing up, we have presented experimental results of negative refraction through a parallel face slab of ETMs made by stacking subwavelength hole arrays. We have carried out a deep analysis based on the simulation of twodimensional dispersion diagram, time magnetic field $H_{y}$ evolution, and large amount of measured data to prove the negative refraction under oblique $P$-polarization incidence in this structure. The measured beam displacements expected by simulation according to our dispersion diagram show good qualitative agreement with previous analytical studies. $^{20,22}$ Moreover, a leaky-wave-based discussion to explain the behavior has been as well introduced.

This work opens the possibility to negative refraction in the optical range using similar structures. ${ }^{27}$

This work was supported by the Spanish Government under contract Consolider "Engineering Metamaterials," through Contract No. CSD2008-00066.
${ }^{1}$ V. G. Veselago, Sov. Phys. Usp. 10, 509 (1968).

${ }^{2}$ J. B. Pendry, Phys. Rev. Lett. 85, 3966 (2000).

${ }^{3}$ R. A. Shelby, D. R. Smith, and S. Schultz, Science 292, 77 (2001).

${ }^{4}$ J. B. Pendry, A. J. Holden, W. J. Stewart, and I. Youngs, Phys. Rev. Lett. 76, 4773 (1996).

${ }^{5}$ J. B. Pendry, A. J. Holden, D. J. Robbins, and W. J. Stewart, IEEE Trans. Microwave Theory Tech. 47, 2075 (1999).

${ }^{6}$ M. Notomi, Opt. Quantum Electron. 34, 133 (2002).

${ }^{7}$ S. Enoch, G. Tayeb, and B. Gralak, IEEE Trans. Antennas Propag. 51, 2659 (2003).

${ }^{8}$ T. W. Ebbesen, H. J. Lezec, H. Ghaemi, T. Thio, and P. A. Wolf, Nature (London) 391, 667 (1998).

${ }^{9}$ L. Martín-Moreno, F. J. García-Vidal, H. J. Lezec, K. M. Pellerin, T. Thio, J. B. Pendry, and T. W. Ebbesen, Phys. Rev. Lett. 86, 1114 (2001).

${ }^{10}$ M. Beruete, M. Sorolla, I. Campillo, J. S. Dolado, L. MartínMoreno, J. Bravo-Abad, and F. J. García-Vidal, Opt. Lett. 29, 2500 (2004).

${ }^{11}$ J. B. Pendry, L. Martín-Moreno, and F. J. García-Vidal, Science
305, 847 (2004)

${ }^{12}$ V. Lomakin and E. Michielssen, Phys. Rev. B 71, 235117 (2005).

${ }^{13}$ M. Beruete, M. Sorolla, and I. Campillo, Opt. Express 14, 5445 (2006).

${ }^{14}$ S. Zhang, W. Fan, N. C. Panoiu, K. J. Malloy, R. M. Osgood, and S. R. J. Brueck, Phys. Rev. Lett. 95, 137404 (2005).

${ }^{15} \mathrm{G}$. Dolling, C. Enkrich, M. Wegener, C. M. Soukoulis, and S. Linden, Science 312, 892 (2006).

${ }^{16}$ A. J. Hoffman, L. Alekseyev, S. S. Howard, K. J. Franz, D. Wasserman, V. A. Podolskiy, E. E. Narimanov, D. L. Sivco, and C. Gmachl, Nature Mater. 6, 946 (2007).

${ }^{17}$ M. Beruete, I. Campillo, M. Navarro-Cía, F. Falcone, and M. Sorolla Ayza, IEEE Trans. Antennas Propag. 55, 1514 (2007).

${ }^{18}$ M. Beruete, M. Sorolla, M. Navarro-Cía, F. Falcone, I. Campillo, and V. Lomakin, Opt. Express 15, 1107 (2007).

${ }^{19}$ M. Navarro-Cía, M. Beruete, M. Sorolla, and I. Campillo, Opt. Express 16, 560 (2008).

${ }^{20}$ A. Mary, S. G. Rodrigo, F. J. Garcia-Vidal, and L. MartinMoreno, Phys. Rev. Lett. 101, 103902 (2008). 
${ }^{21}$ P. F. Goldsmith, Quasioptical Systems-Gaussian Beam, Quasioptical Propagation, and Applications (IEEE, New York, 1998).

${ }^{22}$ F. J. García de Abajo, R. Gómez-Medina, and J. J. Sáenz, Phys. Rev. E 72, 016608 (2005).

${ }^{23}$ www.cst.com

${ }^{24}$ P. Yeh, J. Opt. Soc. Am. 69, 742 (1979).

${ }^{25}$ T. Li, H. Liu, F. M. Wang, Z. G. Dong, S. N. Zhu, and X. Zhang,
Opt. Express 14, 11155 (2006).

${ }^{26}$ A. Mary, S. G. Rodrigo, L. Martín-Moreno, and F. J. GarcíaVidal, J. Phys.: Condens. Matter 20, 304215 (2008).

${ }^{27}$ Z. H. Tang, R. W. Peng, Z. Wang, X. Wu, Y. J. Bao, Q. J. Wang, Z. J. Zhang, W. H. Sun, and Mu. Wang, Phys. Rev. B 76, 195405 (2007). 